\title{
Fortifying the Island at the time of the viceroy Ferrante Gonzaga (1536-1546): sites, master builders and designers, clients
} Emanuela Garofalo

Università degli Studi di Palermo, Scuola Politecnica, Dipartimento di Architettura, Palermo, Italy, emanuela.garofalo@unipa.it

\begin{abstract}
The ten-year government of Ferrante Gonzaga marks a crucial time in the process of modernization of fortifications on the largest island of the Western Mediterranean. Even if the cornerstones of the strategy pursued and the projects undertaken to realize an adequate defense system have already been highlighted by previous studies, and it is well-known how much the viceroy himself and the royal engineer Antonio Ferramolino participated in resolving the longstanding problem, new interesting data emerge from research on the archival collections of Tribunale del Real Patrimonio, kept in the State Archive of Palermo. Using this mostly unpublished documentation, the paper focus on building sites started during this decade, looking at aspects related to construction as well as to organization and functioning, and on the intertwining of skills and responsibilities of the various actors involved in the various phases, from design to execution. The information included in the documents allows us to clarify the state of the art of fortification works undertaken in the main Sicilian cities during that decade, providing technical and financial data, but also information on the measures that were adopted to guarantee efficiency and promptness on the sites. Moreover, this knowledge forms a good basis for a new analysis of the roles played by the different people involved.
\end{abstract}

Keywords: Ferrante Gonzaga, Antonio Ferramolino, Sicily, building sites.

\section{Introduzione}

Il decennio di governo di Ferrante Gonzaga (1536-1546) in qualità di viceré di Sicilia si colloca in un momento cruciale dello scontro politico-militare tra Carlo $\mathrm{V}$ e Solimano il Magnifico, dal 1536 alleato con i Francesi. Altrettanto cruciale è il ruolo assegnato all'isola nella strategia militare imperiale, come presidio difensivo avanzato nel Mediterraneo (Giarrizzo, 1989; Giuffrida, 1999). La particolare congiuntura storico-politica e le esigenze che ne derivavano, quindi, hanno sicuramente giocato un ruolo determinante nella scelta di conferire il prestigioso incarico di viceré di Sicilia a un uomo d'armi e fedelissimo dell'imperatore come Gonzaga (Gosellini, 1574; Capasso, 1905-1906), giustificando inoltre la centralità della questione difesa nell'azione di governo di quest'ultimo, nonché la concentrazione di sforzi finanziari e progettuali sull'ammodernamento e messa a sistema delle fortificazioni dell'isola.

Occorre a questo punto precisare che non si trattava tuttavia di partire da zero, trovando l'azione promossa da Gonzaga un importante precedente nel programma di interventi e nei cantieri avviati dal suo predecessore, Ettore Pignatelli duca di Monteleone (Vesco, 20092010). Allo stesso modo, sulle solide basi poste da Gonzaga, spetterà al suo successore Juan de Vega portare a termine la difficile impresa, introducendo diverse novità. Il dispendioso e non sempre lineare processo di 
ammodernamento e implementazione delle difese dell'isola al principio dell'età moderna è quindi frutto dell'intreccio delle visioni strategiche e progettuali portate avanti, come in una ideale staffetta, dai tre viceré e da altrettanti ingegneri militari di nomina imperiale (Pietro Antonio Tomasello, Antonio Ferramolino e Pedro Prado) succedutisi nel delicato incarico quasi con la stessa cadenza dei primi (Giuffrida, 2007).

L'importanza strategica e la mole delle opere intraprese pressoché simultaneamente in diversi capisaldi del territorio siciliano negli anni del Gonzaga, nonché la modernità delle strutture progettate, è comunque innegabile, così come è a nostro avviso la centralità della visione strategica complessiva dallo stesso brevemente delineata, in particolare nelle due note relazioni indirizzate all'imperatore nel 1537 e a fine mandato, nel 1546 (Dufour, 1999). Tali documenti restano le fonti primarie per conoscere il pensiero del viceré sulla questione, con alcuni ripensamenti tra la prima e la seconda relazione.

\subsection{La difesa dell'isola nella Relazione delle cose di Sicilia (1546)}

In sintesi, dopo aver enfaticamente enunciato un cambio di strategia nella valutazione dell'importanza da attribuire alle diverse piazzeforti, nella Relazione delle cose di Sicilia (Gonzaga, ed. 1896) Gonzaga illustra la condizione delle tre coste - di levante, tramontana e mezzogiorno -, lo stato dell'arte al momento del suo arrivo e i cantieri avviati per rimediare alle principali carenze riscontrate. Se trascurabile appariva l'assetto della costa meridionale, «per non avere alcun ridotto nel quale Armata possa firmarsi» e per l'assenza di fortezze delle quali il nemico potesse impossessarsi, sulle altre due si erano concentratati tutti gli sforzi.

Lungo la costa settentrionale, protetta comunque dalla presenza di «montagne asprissime» a ridosso della stessa, si era posta particolare cura al completamento delle strutture difensive di Trapani, «fortificata dalla parte di terra molto bene, ma dalla parte di mare apertissima», e di
Milazzo, nonché a un complessivo ripensamento del sistema difensivo di Palermo «nella quale non era cosa di buono». Relativamente a quest'ultima, Gonzaga si attribuisce il merito di averla dotata di un fitto circuito di bastioni, nonché di aver escogitato un astuto espediente per accelerare il processo di realizzazione del fossato mancante.

Le principali preoccupazioni erano state destate infine dalla costa orientale «come quella, che dava più facile adito, $[\ldots]$ per essere dotata di bellissimi porti, di Città principali, per essere paese fertilissimo et piano». Qui, al soddisfacente assetto riscontrato a Siracusa si contrapponeva l'abbandono nel quale versavano le strutture difensive a Messina e a Catania, nonché l'indifendibile porto di Augusta. A tali criticità si era posto rimedio avviando innanzitutto un'alacre attività costruttiva per la difesa di Messina, dal momento che «quella occupata, si può dire che fusse occupata la chiave di esso (regno) et del regno di Napoli». L'importanza strategico-militare attribuita dal Gonzaga a Messina emergeva già nella precedente relazione del 1537.

Relativamente alla città di Catania, invece, se nel documento del 1537 la valutava indifendibile e da abbandonare in caso di invasione, tale convincimento mutò radicalmente negli anni successivi, come appare evidente nella relazione del 1546. La ricchezza del sito e la possibilità di sopperire alle carenze del porto per la vicinanza di quello di Augusta, nonché l'impegno preso dalla città a finanziare l'impresa con 40.000 scudi - oltre alle pressioni esercitate dall'élite locale - lo avevano persuaso ad avviare un progetto di fortificazione, portato «in assai buon termine» al momento della sua partenza.

Procedendo verso sud, si presentava poi la spinosa questione del porto di Augusta «il qual per esser tanto grande che più tosto si può chiamar Golfo». Per le difficoltà e l'eccessiva spesa che avrebbe richiesto l'attuazione di un programma di fortificazione, la risoluzione presa era stata di puntare sul potenziamento delle strutture difensive delle tre città esistenti intorno al grande porto, Catania e Siracusa, a nord e a sud, Lentini un po' arretrata in posizione 
centrale. In merito a quest'ultima, all'iniziale idea di fortificare una parte dell'abitato si era sostituita, per i guasti causati dal terremoto del 1542, l'ipotesi di una ricostruzione complessiva della città in altro sito, questione in realtà ancora irrisolta al momento della partenza di Gonzaga dall'isola e che avrà un diverso epilogo con la fondazione di Carlentini negli anni di De Vega (Aricò, 2012).

Per Siracusa, infine, sebbene proponesse un restringimento della fortezza - riferendosi forse alle strutture all'ingresso della città dalla parte del Forte Casanova (Dufour, 1999) - la reputava «forte nel modo che la sta», avendo promosso come ulteriore precauzione per la sicurezza dei territori limitrofi e a copertura della stessa Siracusa la fortificazione della città di Noto, nell'immediato entroterra.

\section{Lo stato dell'arte attraverso le Lettere Viceregie}

Se la relazione del 1546 fornisce quindi un efficace quadro d'insieme delle problematiche affrontate e delle strategie perseguite, utili precisazioni sullo svolgimento del programma, la sequenza delle opere e gli obiettivi raggiunti si ricavano dal corpus delle Lettere Viceregie relative al governo di Gonzaga, fino ad oggi solo parzialmente indagato ${ }^{2}$.

Nel 1537 si concentra innanzitutto una serie di disposizioni relative alla città di Messina, dove alle riparazioni, innalzamento di mura e parapetto nel preesistente castello del Salvatore ancora sulla scorta delle disposizioni lasciate dall'ingegnere Tomasello - e in quello di Matagrifone, si aggiunge la costruzione di due nuovi bastioni, della "cardilla" e nella torre di Don Blasco. Ai lavori in atto nelle fortezze di Messina si riferiscono inoltre le disposizioni contenute in diverse lettere, tra 1540 e 1546, per lo più inerenti l'erogazione dei fondi necessari alla prosecuzione delle fabbriche, confermando nel complesso la centralità attribuita alla città nel più generale progetto di difesa del regno.

Nello stesso arco cronologico, alcune lettere testimoniano di lavori nel castello e nelle mura di Milazzo, compresa la costruzione di un bastione secondo il progetto dell'ingegnere Ferramolino e le opere per «voltari rustici lu dammuso di la porta di lisola» ${ }^{3}$. Dal 1542 compaiono notizie circa le opere di fortificazione nella città di Catania, dagli accordi per la spesa di 40.000 scudi erogati dalla stessa città, all'approntamento di disegni da parte di Ferramolino, all'invio di un adeguato numero di pirriaturi (maestri cavatori) per la costruzione di un baluardo. Oltre che per Catania, dalla stessa lettera si apprende che Ferramolino aveva contemporaneamente realizzato disegni per le fortificazioni di Lentini e di Noto. Relativamente a quest'ultima, si segnala la presenza in uno dei volumi dell'«Instructione et ordinatione di quanto si havirà di exequiri per li magnifici deputati eletti a la cura di li fabrichi», emanata dallo stesso Gonzaga il 31 maggio del 1542, che osserveremo più dettaglio di seguito.

Le ingenti opere avviate a Palermo, sono invece citate soltanto in una lettera del 31 dicembre 1541, da Gonzaga indirizzata a Ferramolino, nella quale lamentando i lenti progressi delle fabbriche auspicava che dalla presenza dello stesso in cantiere derivasse una accelerazione.

Due lettere affrontano la questione della costruzione di una torre a difesa del caricatore di Agrigento, questione per la risoluzione della quale il viceré aveva inviato nel marzo del 1543 l'ingegnere Ferramolino, col mandato di fornire disegni e istruzioni, verificando inoltre il prezzo fissato per l'acquisto di calce e pietra e per la manodopera. L'opera in realtà era in agenda già da tempo, come testimonia tra l'altro una supplica indirizzata al viceré il 12 dicembre del 1541. Sull'argomento Gonzaga tornerà nel maggio del 1546, per rendere esecutive le disposizioni provenienti dallo stesso imperatore, su sollecitazione dell'Università di Agrigento.

Per imprimere una accelerazione ai cantieri avviati, infine, il viceré aveva in più occasioni vietato che i maestri e gli stessi materiali da costruzione - calce e pietra potessero essere distratti per qualsiasi altra opera fino al compimento dei primi, disponendo talora anche il reclutamento coatto di maestranze dai centri limitrofi. 


\section{Dal progetto all'esecuzione, i soggetti coinvolti e le competenze}

Oltre alle indicazioni sulle opere avviate e sul loro avanzamento, dalle Lettere Viceregie è possibile ricavare alcune informazioni su diversi soggetti convolti nei cantieri delle fortificazioni.

Figura chiave, tanto per la messa in atto del generale disegno difensivo, quanto per l'effettivo avanzamento delle opere è di certo l'ingegnere regio Antonio Ferramolino. Dalle Lettere Viceregie, innanzitutto, emerge a pieno la poliedrica e itinerante attività dell'ingegnere, chiamato a: redigere disegni di progetto; dettare le odinationi, ossia dettagliate istruzioni per poter procedere all'esecuzione delle opere anche in sua assenza; vigilare sull'andamento dei cantieri con periodiche ispezioni e permanenze nei diversi siti, a garanzia non soltanto di una buona qualità e di una celere realizzazione delle opere programmate, ma anche di una corretta gestione tanto dei materiali e delle maestranze, quanto dell'assegnazione degli appalti (staglio); a selezionare, infine, le maestranze da inviare nelle postazioni nord-africane ${ }^{4}$.

Le stesse lettere inoltre danno contezza del rapporto di fiducia da questi instaurato con il viceré, che in più occasioni non lesina attestati di stima, elogiandolo per il servizio reso e intimando di «prestare quella credenza al magnifico Ferramolino come alla persona nostra propria ${ }^{5}$. Spostamenti e permanenze dell'ingegnere nei diversi cantieri non sembrano rispondere a criteri prefissati ma alle esigenze contingenti, essendo spesso regolati da direttive provenienti dallo stesso viceré, in funzione dello stato di avanzamento delle fabbriche o di problematiche relative alla gestione delle opere. Così, ad esempio, in una lettera del 10 dicembre 1541 Gonzaga preannuncia ai deputati delle fabbriche e ai giurati della città di Messina di avere convocato l'ingegnere «per dare ordine a la expeditione di li fabrichi come per li altri cosi che occurressero farse per la bona tutela et defensione de ditta cita» ${ }^{6}$; a preoccupazioni di diversa natura si deve invece il suo invio a Catania, per vigilare sulla fornitura di calce e sull'assegnazione dello staglio (appalto per la costruzione), come precisa una lettera del 20 marzo 1542; nella stessa data, inoltre, Gonzaga informa i giurati e i deputati delle fabbriche di Noto di avere ordinato a Ferramolino di recarsi mensilmente nella città per sorvegliare l'andamento del cantiere.

Sebbene la componente retorica non vada trascurata, nelle lettere si fa più volte riferimento a disposizioni impartite dallo stesso Gonzaga per i singoli cantiere che sembrerebbero confermare la sua diretta partecipazione alle scelte progettuali e operative. Il caso più eloquente è quello relativo alle fortificazioni della città di Messina, per le quali il viceré precisa in una lettera rivolta ai giurati della città il 23 agosto del 1540 che si doveva procedere «secundo lo novo modello che per noi è stato ordinato» ${ }^{7}$.

In aggiunta al binomio Gonzaga/Ferramolino, dalle lettere emerge - sebbene in modo episodico - l'interessamento di altri soggetti alle vicende relative alla difesa dell'isola, che aprono uno spiraglio sulla maggiore articolazione di responsabilità che un programma così complesso richiedeva. Nel febbraio del 1540, il marchese di Terranova, che in qualità di Presidente del Regno svolgeva in tale frangente le veci del viceré assente, dispone una retribuzione di dieci scudi in favore di Antonio di Milazzo «ingigneri di Sua Maestà Cesarea in questo regno», per «aiuto di costa», ma anche per il servizio prestato nella città di Messina ${ }^{8}$. Alle valutazioni del capomastro delle fabbriche Domenico Lanza si affida lo stesso per una delicata decisione tecnica relativa alla costruzione di una volta in corrispondenza di una porta nelle fortificazioni di Milazzo. Al parere espresso dallo «spettabili regio consigleri et nostro consultore» Andrea Arduino (Bazzano, 2009) fa riferimento invece Gonzaga in una lettera del 18 gennaio 1542, per le riparazioni intraprese nel bastione di Don Blasco a Messina ${ }^{9}$. Compiti di supervisione sono assegnati, infine, rispettivamente a Don Pedro Gonzales de Mendoza, nella primavera del 1543 per le fortificazioni di Siracusa e Messina, e a Don Carlo Elabes, chiamato a risiedere a Messina nel marzo del 1546 per sovrintendere alle fortificazioni di quest'ultima e della vicina città di Milazzo. 


\section{Il cantiere, dati tecnici e organizzazione}

Scendendo di scala, un secondo aspetto sul quale intendiamo soffermarci è quello relativo al cantiere, cioè alla costruzione delle singole strutture difensive, osservandone organizzazione e aspetti tecnici attraverso la documentazione di diversa natura - rintracciata per due capisaldi del complessivo progetto di difesa perseguito nel decennio in esame, Noto e Palermo.

\subsection{I capitoli per le fortificazioni di Noto (1542)}

Come accennato in precedenza, tra le Lettere Viceregie sono registrati i capitoli emessi dal viceré Gonzaga per la costruzione delle fortificazioni di Noto, il 31 maggio del 1542. Articolato in sedici punti, il documento fornisce innanzitutto una chiara testimonianza dell'organizzazione amministrativa preposta a vigilare sul corretto funzionamento del cantiere. Dallo stesso viceré era stato designato un «depositario et pagatore di ipsi fabrichi», al quale $i$ giurati dovevano far pervenire annualmente la somma di denaro che la città doveva destinare alle fortificazioni.

Le questioni più tecniche, legate in particolare alla fornitura dei materiali da costruzione e al reclutamento delle maestranze, erano di competenza di due deputati, che duravano in carica soltanto due mesi. Altri due ufficiali, prescelti ancora dal viceré, completavano l'organigramma il «tenitore di lo libro di li fabrichi», responsabile cioè della compilazione di un registro di fabbrica, e il «rechipituri di li attratti», ossia un funzionario preposto a ricevere e ispezionare tutti i materiali acquistati per la costruzione.

Gli obblighi strettamente definiti per ciascuna di queste figure e il sistema di controlli incrociati istituito miravano a scongiurare il pericolo di frodi. Così, ad esempio, se tenitori e richipituri erano tenuti ad annotare nel libro di fabbrica $i$ nominativi di tutti i maestri e manovali impiegati a giornata nel cantiere, i deputati erano a loro volta obbligati a revisionare quotidianamente tali annotazioni «per non si posse commettere alcuna fraude». I capitoli disponevano inoltre che i suddetti officiali si riunissero ogni quindici giorni per discutere delle necessità della fabbrica, man mano che questa avanzava.

Oltre agli aspetti amministrativi brevemente commentati, i capitoli affrontano questioni pratiche inerenti $\mathrm{i}$ materiali da costruzione (calce, sabbia e pietra) e le maestranze da coinvolgere nel cantiere. Relativamente ai primi, si precisa in particolare che la calce doveva essere di buona qualità, ben cotta e senza pietre, dovendo provvedere al momento della consegna a bagnarla e ricoprirla di sabbia. Per la fornitura di pietra, il più possibile abbondante, si richiede di estrarla direttamente nel sito sul quale insisteva la nuova costruzione ${ }^{10}$, facendo in tal modo economia sui costi di trasporto. In aggiunta alla pietra ottenuta per tal via, si disponeva l'acquisto di «cento cantoni di longhezza di palmi quattro et di larghezza per omni verso di palmi dui quali diviranno di serviri a li canthoneri et bombarderi», cioè elementi lapidei accuratamente intagliati da utilizzare per la realizzazione delle parti della struttura chiamate ad avere una maggiore resistenza. La sabbia, infine, doveva essere acquistata in un quantitativo ingente ancor prima dell'avvio dei lavori, provvedendo anche in seguito a rifornire continuamente il cantiere. Relativamente alle maestranze da coinvolgere, si prescrive l'ingaggio di «alcuni boni perriaturi et alcuni calcarari» (cioè cavatori ed esperti nel confezionamento della calce), da reclutare principalmente nella contea di Modica.

La maggior rapidità dei lavori garantita dal sistema condiziona infine la scelta di affidare a staglio (cioè in appalto) piuttosto che a giornata tanto l'esecuzione dei lavori di muratura, «quanto lo fare di la petra et di la calche», «advertendo peroche ditta opera di staglo si fazi et perfecta et non si permetta che si fazi trista per farsene multa quantita».

\subsection{La costruzione delle fortificazioni di Palermo in due libri contabili (1536-1539)}

L'annotazione quotidiana di tutte le spese sostenute, ivi compresi i pagamenti ai diversi operatori che a vario titolo avevano prestato servizio nel cantiere, imposta dai capitoli per le fortificazioni di Noto era in effetti una prassi 
generalizzata. Strumento di controllo di una regolare conduzione del cantiere, a tutela del bene collettivo, tali registri lamentabilmente sono andati dispersi. Un'eccezione è costituita da due volumi che riportano la contabilità relativa ai lavori per la costruzione delle fortificazioni di Palermo, tra il 12 novembre 1536 e il 23 agosto $1539^{11}$. All'esigua lista degli introiti riservati a finanziare tali opere, essenzialmente provenienti dalla gabella della farina, riscossa in città da due appositi deputati, segue la dettagliata annotazione di tutte le spese sostenute, de die in diem, per l'esecuzione delle stesse.

I due volumi fotografano l'avvio della messa in opera del progetto per le fortificazioni di Palermo ideato da Ferramolino (Di Giovanni, 1896; Giuffrè, 1976; Tadini, 1977; Palazzolo, 2007) che oltre alla costruzione di nuovi bastioni prevedeva significative demolizioni di strutture preesistenti, talora anche di recente costruzione. Il processo di ammodernamento del circuito difensivo della capitale dell'isola così avviato avrà tuttavia tempi di esecuzione dilatati, con significative modifiche al disegno iniziale. Oltre che sul progetto in corso di esecuzione concentrato in particolare in quegli anni sulla costruzione dei bastioni della Spasimo e di torre tonda - i due volumi contabili forniscono preziose informazioni per la conoscenza del cantiere.

Dai pagamenti indirizzati a maestri, maestranze e fornitori si ricava innanzitutto un quadro delle professionalità coinvolte, dei materiali e degli arnesi da lavoro utilizzati, nonché delle macchine da cantiere predisposte. Le diverse attività via via retribuite restituiscono inoltre la sequenza delle operazioni necessarie per l'attuazione del progetto. I perriaturi (cavatori) provvedevano innanzitutto all'escavazione dei fossati, liberati dalla terra e dalle pietre ad opera di semplici manovali, via via che i primi procedevano nello scavo. L'operazione quindi aveva una duplice utilità, garantendo oltre alla realizzazione del fossato un buon approvvigionamento di materiali da costruzione in situ. Ancora all'insegna dell'ottimizzazione delle risorse e delle energie lavorative impiegate appare inoltre l'ulteriore acquisizione di pietra per le nuove fabbriche dalle strutture difensive che si era deciso di dismettere (torri medievali e rivellini), alla cui demolizione si procedeva parallelamente. Tra queste figurano anche quattro torri del palazzo reale. Oltre alla pietra, l'approvvigionamento di materiali riguarda principalmente calce e soprattutto sabbia. In misura minore, tra gli acquisti compaiono anche tavole, travetti e altri elementi lignei, utilizzati per lo più per costruire scale o strutture a supporto di macchine da cantiere. Tra queste figurano gru e trabucchi, impiegati principalmente per il sollevamento dei materiali provenienti dallo scavo dei fossati. Tra gli arnesi da lavoro acquistati figurano a più riprese zappe e zappuni, marruggi e pali di ferro «per calcare la pietra». Procedendo nell'attività di costruzione delle nuove strutture, le professionalità coinvolte includono maestri muratori (o fabricatores), come Antonio di Baudo e Giovan Battista Faya, e intagliatori coinvolti in particolare per la realizzazione di «merguloni et cannoneri». Per la realizzazione delle macchine da cantiere, di scale in legno e di centine (furmi) per la costruzione di volte, si registra inoltre la presenza di maestri d'ascia. Tra le diverse professionalità annotate nei registri in esame, segnaliamo infine il pittore trapanese Francesco Soprano compensato per aver «depinto la pianta di quista chitati cum soi misuri per la fortificationi». Il conferimento di un simile incarico, preventivo all'avvio di una riforma urbana importante, a un pittore conta del resto nella Sicilia del XVI secolo altri esempi (Gazzè, 2012).

Le retribuzioni indirizzate ai diversi soggetti coinvolti nella vicenda costruttiva rivelano inoltre alcuni aspetti relativamente all'organizzazione e al controllo di questi complessi cantieri pubblici. Come già sottolineato, la figura di vertice è quella dell'ingegnere, progettista e autore delle ordinazioni, ossia disposizioni esecutive per l'attuazione del progetto. Nel caso in esame, si apprende che tali ordinazioni furono copiate in tre esemplari distribuiti ai tre deputati delle fabbriche - uno eletto dalla regia curia e due dal consiglio civico tra i procuratori della città - per potere dare ordini circa le opere da eseguire, 
garantendo la fedeltà al progetto dell'ingegnere. Come nel caso di Noto, la supervisione amministrativa era affidata alla vigilanza esercitata dai municioneri (equivalenti al tenituri del libro), chiamati ad annotare quotidianamente in appositi registri i nominativi dei lavoratori impegnati nel cantiere e l'attratto. Sopra i munizionieri stavano i deputati e a questi si affiancano altri funzionari, cioè il tesoriere e il secreto, per gli aspetti finanziari.

Se l'ingegnere è il principale responsabile del progetto generale e fornisce le istruzioni attuative, la sua saltuaria presenza in cantiere rendeva necessaria l'introduzione di una figura stabilmente alla guida della costruzione.

La corretta esecuzione delle opere era quindi garantita da un capomastro delle fabbriche, nel caso specifico Antonio Belguardo sostituito poi da Francesco Basilicata, al quale veniva corrisposto per tale incarico uno stipendio annuo di 30 onze. Se la stabilità dell'incarico consentiva al capomastro di incidere sull'esito finale della costruzione, i gradi di libertà di quest'ultimo rispetto alle previsioni di progetto dovevano comunque essere limitati.

Una conferma in tal senso proviene da un rimborso spese accordato a Belguardo e annotato nei registri del cantiere palermitano, «per haviri andato in trapani per conferiri cum lu ingigneri supra la maramma di la turri di lo tronu».

I documenti indicano nel caso in esame anche l'esistenza di un capo dei manovali - tale Gabriele Blanchino - che riceveva i pagamenti che a sua volta distribuiva agli operai dallo stesso controllati. Questi risulta inoltre coadiuvato da due aiutanti rispettivamente addetti alla supervisione del lavoro dei manovali - tale Stefano Russo - e alla ricezione della sabbia trasportata via mare fino al piano di San Sebastiano.

\section{Conclusioni}

I documenti commentati contribuiscono a tracciare uno spaccato, ancora provvisorio, ma denso di spunti per una conoscenza più approfondita dei cantieri per la costruzione delle fortificazioni in Sicilia negli anni del governo di Ferrante Gonzaga.

Sebbene molti aspetti, in particolare quelli relativi alle responsabilità progettuali meriterebbero una più approfondita riflessione caso per caso, nel quadro generale e attraverso gli esempi esaminati, emerge con chiarezza l'esistenza di un modello organizzativo ben preciso e replicabile, e l'attenzione alla costituzione di un organigramma piramidale piuttosto articolato, ma in grado di garantire efficienza, rapidità, qualità delle opere, ma anche il controllo della gestione finanziaria.

\section{Note}

(1) The research leading to these results has received funding from the European Research Council under the European Union's Seventh Framework Programme (FP7/2007-2013) / ERC grant agreement $n^{\circ} 295960$ - COSMED.

(2) Archivio di Stato di Palermo (da ora ASP), Tribunale del Real Patrimonio (da ora TRP), Lettere Viceregie, voll. 299-347.

(3) ASP, TRP, Lettere Viceregie, vol. 339, cc. $493 \mathrm{v}-497 \mathrm{v}$.

(4) Si tratta delle fortezze di Bugia e Goletta, per le quali Ferramolino è incaricato di selezionare una squadra composta da un capomastro, cinque muratori e quattro carcaroti (maestri che confezionavano la calce). ASP, TRP, Lettere Viceregie, vol. 327, cc. 86v-87r.

(5) Ivi, vol. 332, c. 493v-49188v.

(6) Ivi, vol. 326, cc. 78r-v.

(7) Ivi, vol. 320, cc. 317r-319r.

(6) Ivi, vol. 321, cc. 364r-v.

(9) Ivi, vol. 327, c. 97v.

(10) Tale soluzione, era stata già attuata in altri cantieri; in particolare è il caso delle fortificazioni di Palermo, come ricordato dallo stesso Gonzaga nella Relazione del 1546.

(11) ASP, TRP, Numerazione Provvisoria, voll. 1336 e 1450 


\section{References}

Aricò N. (2012). "Pedro Padro e la fondazione di Carlentini" in Fondazioni urbane. Città nuove europee dal medioevo al Novecento, a cura di Casamento A. Kappa. Roma, pp. 167-208.

Bazzano N. (2009). "La Sicilia di Ferrante Gonzaga (1535-1543): uno schizzo storiografico" in Ferrante Gonzaga. Il Mediterraneo, l'Impero (1507-1557), Atti del Convegno di Studi (Guastalla, 5-6 ottobre 2007), a cura di Signorotto G. Bulzoni Editore. Roma, pp. 119-137.

Capasso G. (1905-1906). "Il governo di Ferrante Gonzaga in Sicilia" in «Archivio Storico Siciliano», XXX, pp. 405-470, e XXXI, pp. 1-112 e 337-461.

Di Giovanni V. (1896). Le fortificazioni di Palermo nel secolo XVI secolo giusta l'ordini dell'ing. Antonio Ferramolino in Documenti per servire alla storia di Sicilia, s. IV, IV. Tipografia "Lo Statuto". Palermo.

Dufour L. (1999). "Città e fortificazioni in Sicilia nell'età di Carlo V" in Vincenzo degli Azani da Pavia e la cultura figurativa in Sicilia nell'età di Carlo V, a cura di T. Viscuso. Ediprint. Siracusa, pp. 1124.

Gazzè L. (2012). Governare il territorio: la Sicilia descritta, misurata, disegnata (secoli XVI-XVII). Bonanno. Acireale-Roma.

Giarrizzo G. (1989). "La Sicilia dal Cinquecento all’Unità d'Italia" in La Sicilia dal Vespro all'Unità d'Italia, a cura di D'Alessandro V., Giarrizzo G. UTET. Torino, pp. 172-192.

Giuffrè M. (1976). "Palermo 'città murata' dal XVI al XIX secolo" in «Quaderno I.D.A.U.», 8, pp. 4168.

Giuffrida A. (1999). La finanza pubblica nella Sicilia del '500. Salvatore Sciascia Editore. Caltanissetta-Roma, pp. 17-19.

Giuffrida A. (2007). "La fortezza indifesa e il progetto del Vega per una ristrutturazione del sistema difensivo siciliano" in Mediterraneo in armi (secc. XV-XVIII), a cura di Cancilia R. Associazione Mediterranea. Palermo, pp. 227-288.

Gonzaga F. (1896). Relazione delle cose di Sicilia fatta da D. Ferrando Gonzaga all'imperatore Carlo $V$ 1546, ed. a cura di Carreri F. C. in Documenti per servire alla storia di Sicilia, s. IV, IV. Tipografia "Lo Statuto". Palermo, pp. 5-27

Gosellini G. (1574). Vita del prencipe don Ferrando Gonzaga. Milano.

Palazzolo A. (2007). Le difese del Gonzaga a Palermo (1535-1546). ISSPE. Palermo.

Tadini G. (1977). Ferramolino da Bergamo: l'ingegnere militare che nel '500 fortificò la Sicilia. Poligrafiche Bolis. Bergamo.

Vesco M. (2009-2010). "Pietro Antonio Tomasello de Padua: un ingeniero militar véneto en la Sicilia de Carlos V" in «Espacio, Tiempo y Forma, Historia del Arte», 22-23, pp. 45-73. 